\title{
Properties of GH4169 Superalloy Characterized by Nonlinear Ultrasonic Waves
}

\author{
Hongjuan Yan, ${ }^{1,2}$ Chunguang Xu, ${ }^{1}$ Dingguo Xiao, ${ }^{1}$ and Haichao Cai ${ }^{1}$ \\ ${ }^{1}$ School of Mechanical Engineering, Beijing Institute of Technology, Beijing 100081, China \\ ${ }^{2}$ College of Mechanical and Materials Engineering, North China University of Technology, Beijing 100144, China
}

Correspondence should be addressed to Hongjuan Yan; yanhongj@sina.com

Received 26 December 2014; Revised 28 January 2015; Accepted 28 January 2015

Academic Editor: Jainagesh A. Sekhar

Copyright (C) 2015 Hongjuan Yan et al. This is an open access article distributed under the Creative Commons Attribution License, which permits unrestricted use, distribution, and reproduction in any medium, provided the original work is properly cited.

The nonlinear wave motion equation is solved by the perturbation method. The nonlinear ultrasonic coefficients $\beta$ and $\delta$ are related to the fundamental and harmonic amplitudes. The nonlinear ultrasonic testing system is used to detect received signals during tensile testing and bending fatigue testing of GH4169 superalloy. The results show that the curves of nonlinear ultrasonic parameters as a function of tensile stress or fatigue life are approximately saddle. There are two stages in relationship curves of relative nonlinear coefficients $\beta^{\prime}$ and $\delta^{\prime}$ versus stress and fatigue life. The relative nonlinear coefficients $\beta^{\prime}$ and $\delta^{\prime}$ increase with tensile stress when tensile stress is lower than $65.8 \%$ of the yield strength, and they decrease with tensile stress when tensile stress is higher than $65.8 \%$ of the yield strength. The nonlinear coefficients have the extreme values at $53.3 \%$ of fatigue life. For the second order relative nonlinear coefficient $\beta^{\prime}$, there is good agreement between the experimental data and the comprehensive model. For the third order relative nonlinear coefficient $\delta^{\prime}$, however, the experiment data does not accord with the theoretical model.

\section{Introduction}

GH4169 superalloy has good integrate performance in 253 $400^{\circ} \mathrm{C}$. It is manufactured as complex components and widely used in aerospace, nuclear energy, petroleum industry, and extrusion dies. Under the tensile loading and the cyclic loading, the metal structural components gradually occur as plastic deformation and induce the damages. They induce the components to crack or fracture.

Fatigue is one of damage mechanisms of components. According to statistics, more than $90 \%$ of the parts failures result from fatigue damages. The fatigue life of structural components can be divided into three stages as follows: early dislocation generation and lattice deformation, microcrack formation, and fracture failure. For structural components, the first and second stages are $80 \% \sim 90 \%$ in fatigue life. Therefore, the detection of early fatigue damage is critical to evaluation of component life [1-3]. The ultrasonic method is believed to be the most promising nondestructive testing technique compared with other destructive and nondestructive testing. Many researches show that the linear ultrasonic parameters such as ultrasonic velocity and attenuation are effective in detection and evaluation in second stage and third stage of fatigue life [4-6]. But the ultrasonic linear parameters are not sensitive to nonlinear behavior and early fatigue state in metal components [5-8]. Therefore it is a focus issue that the nonlinear ultrasonic parameters evaluate the early fatigue state or stress in metal components and predict the fatigue life.

In 1755, Euler proposed the concept of nonlinear acoustic. Researchers such as Lagrange (1760) [9], Stokes (1848) [10], and Rayleigh (1910) [11] studied the theory of nonlinear acoustic [9]. In the 1960s, researchers began to study the phenomenon of nonlinear acoustic in solid [12]. In 1963, Hikata et al. observed that the harmonic waves appeared in aluminum. And Breazeale et al. detected third order elastic constants of single crystal aluminum and copper in 1963 and $1968[13,14]$. Buck et al. studied the acoustic harmonic generation at unbonded interfaces and fatigue cracks [15]. Nagy used nonlinear ultrasonic waves to assess fatigue damage in plastics [16]. Kim et al. used nonlinear ultrasonic waves to characterize fatigue damage in a nickelbase superalloy [17]. Metya et al. studied the generation of second harmonic wave in grade maraging steel [18]. Shui et al. 
used nonlinear longitudinal waves to evaluate plastic damage in metal materials [19]. Ruiz et al. used nonlinear acoustic parameter to assess early detection of thermal degradation of mechanical properties in 2205 duplex stainless steel [20]. Punnose et al. used nonlinear ultrasonic waves to evaluate fatigue in austenitic stainless steel in low cycle regime [21]. The results show that the second order nonlinear coefficient $\beta$ is related to the fatigue life or fatigue damage of materials. But few researchers studied the relationship of the third order nonlinear coefficient versus stress or fatigue life.

The one-dimensional nonlinear ultrasonic wave motion equation is solved by perturbation method. During tensile testing and bending fatigue testing, the relationships of nonlinear ultrasonic parameters and tensile stress or fatigue cycles are studied. The nonlinear ultrasonic testing system is used to detect the received signal. RETIC advanced measurement system generates a tone burst signal and receives the signal propagated in GH4169 superalloy plate. The received signals are transformed by FFT. The fundamental and harmonic amplitudes are obtained, and the parameters are calculated. The relationship curves of nonlinear parameters as the functions of tensile stress or fatigue life are obtained to detect or predict stress or fatigue life of GH4169 superalloy.

\section{Nonlinear Acoustic Properties}

2.1. Nonlinear Acoustic Wave Theory. When one-dimensional longitudinal wave propagates in nonlinear medium, the wave motion equation is as follows [22]:

$$
\frac{1}{\rho} \cdot \frac{\partial \sigma}{\partial x}=\frac{\partial^{2} u}{\partial t^{2}}
$$

where $u$ is the displacement along $x$ direction, $\rho$ is the density of medium, and $\sigma(x, t)$ is the positive stress along $x$ direction. The perturbation method is used to solve (1). The solution is

$$
\begin{aligned}
u(x, t)= & A_{1} \cos (k x-\omega t)-\frac{\beta_{1}}{8} k^{2} A_{1}^{2} x \cos 2(k x-\omega t) \\
& +\frac{\beta_{2}}{24} k^{3} A_{1}^{3} x[\cos 3(k x-\omega t)+3 \cos (k x-\omega t)],
\end{aligned}
$$

where $\omega$ is the circular frequency and $k$ is the wave number. If the amplitude of $\cos 2(k x-\omega t)$ and $\cos 3(k x-\omega t)$ are, respectively, assumed as $A_{2}$ and $A_{3}$, they can be obtained:

$$
\begin{aligned}
& \beta=\frac{8 A_{2}}{k^{2} x A_{1}^{2}}, \\
& \delta=\frac{24 A_{3}}{k^{3} x A_{1}^{3}} .
\end{aligned}
$$

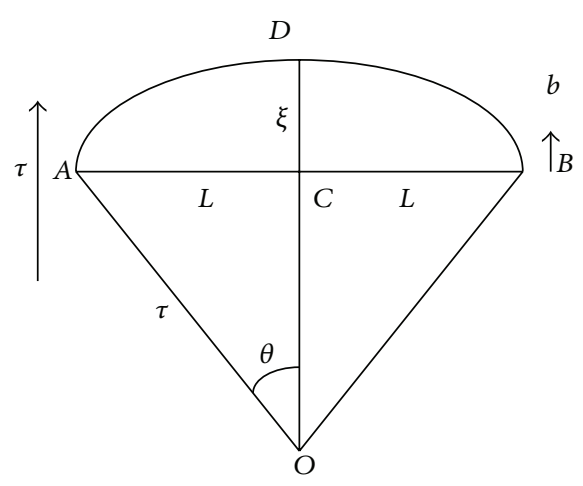

FIGURE 1: Dislocation monopole model.

With the fixed frequency and the fixed propagation distance, the nonlinear coefficients $\beta$ and $\delta$ are, respectively, proportional to $A_{2} / A_{1}^{2}$ and $A_{3} / A_{1}^{3}$. In the measurements, the relative nonlinear parameters can be defined as

$$
\begin{aligned}
& \beta^{\prime}=\frac{A_{2}}{A_{1}^{2}}=\frac{k^{2}}{8} \beta x, \\
& \delta^{\prime}=\frac{A_{3}}{A_{1}^{3}}=\frac{k^{3}}{24} \delta x .
\end{aligned}
$$

The $\beta^{\prime}$ and $\delta^{\prime}$ are the functions of propagation distance $x$. The relationship of $\beta^{\prime}$ and $x$ is a good linearity for propagation distance in the far field. In current experiments, $\beta^{\prime}$ is measured over $x$ [23]. And in the optimized distance, $\beta^{\prime}$ and $\delta^{\prime}$ are measured over stress and fatigue life.

2.2. Dislocation Model for Second Order Nonlinear Coefficient. Nonlinearity of solid material mainly comes from two aspects: (1) nonharmonic property of particles interaction force of the material and (2) internal microdefects of the material, such as crystal dislocation, lattice slip, and microcrack. Many researches show that the ultrasonic nonlinearity induced by dislocations is the main factor leading to the variation of ultrasonic nonlinearity. Cantrell et al. promoted two dislocation models $[15,24,25]$.

Hikata et al. [12], Buck et al. [15], and Cantrell [24] proposed the dislocation monopole model (shown in Figure 1). The generation of the second harmonic wave depends on the motion of the dislocation between the dislocation pinning points. The nonlinear acoustic coefficient is written as [24]

$$
\beta_{m p}=-\frac{E_{3}}{E_{2}}+\frac{24}{5} \cdot \frac{\Omega \Lambda_{m p} L^{4} R^{3} E_{2}^{2}}{G^{3} b^{2}} \sigma,
$$

where $\Lambda_{m p}$ is the dislocation density, $b$ is Burgers vector, $E_{2}$ and $E_{3}$ are, respectively, the second or third order elastic coefficients, $G$ is shear modulus, $\Omega$ is the transform coefficient from shear strain to principle strain, and $R$ is the transform coefficient from shear stress to principle stress.

The first part of (4) is relative to second and third order elastic coefficients $E_{2}$ and $E_{3}$. It is caused by lattice and is 


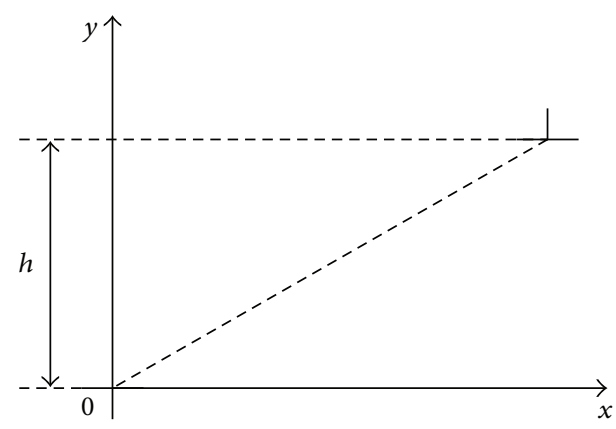

Figure 2: Dislocation dipole model.

negligible in tensile testing and fatigue bending testing. The second part of (4) is relative to dislocation density, string length, and stress. It is caused by dislocation and is the main factor to the ultrasonic coefficient.

Cantrell [24] proposed dislocation dipole model. With the increasing of stress and fatigue state, the dislocations increase and slip and interact. They form the dislocation dipole model (shown in Figure 2). Cash and Cai [25] improved the dipole model and the nonlinear acoustic coefficient is written as

$$
\begin{aligned}
\beta_{d p}= & -\frac{E_{3}}{E_{2}}+\frac{16 \pi^{2} \Omega \Lambda_{d p} h^{3} R^{2}(1-\nu)^{2} E_{2}^{2}}{G^{2} b} \\
& +\frac{384 \pi^{3} \Omega \Lambda_{d p} h^{4} R^{3}(1-\nu)^{3} E_{2}^{2}}{G^{3} b^{2}} \sigma,
\end{aligned}
$$

where $v$ is Poisson ratio and $h$ is the distance of dipoles. The second part of (5) is relative to the dislocation density and the distance of dipoles and is the main factor to the ultrasonic coefficient.

The monopole model and the dipole model have their limits. The two models are, respectively, according to the measurement in some experiments. In the monopole model, the nonlinear coefficient is related to the length of the dislocation string and stress. But in the dipole model, the nonlinear coefficient is related to the dislocation density and the distance of dipoles. Cantrell proposed a comprehensive model [26]. The total nonlinear coefficient is the summation of elastic anharmonicity and the acoustic nonlinearity contributed by the monopole and the dipole, which can be expressed as [26]

$$
\begin{aligned}
\beta= & a_{1} \cdot \frac{24}{5} \cdot \frac{\Omega \Lambda_{m p} L^{4} R^{3} E_{2}^{2}}{G^{3} b^{2}} \sigma+a_{2} \\
& \left(\frac{16 \pi^{2} \Omega \Lambda_{d p} h^{3} R^{2}(1-v)^{2} E_{2}^{2}}{G^{2} b}\right. \\
& \left.+\frac{384 \pi^{3} \Omega \Lambda_{d p} h^{4} R^{3}(1-v)^{3} E_{2}^{2}}{G^{3} b^{2}} \sigma\right),
\end{aligned}
$$

where $a_{1}$ and $a_{2}$ are weight coefficients.
2.3. Dislocation Model for Third Nonlinear Coefficient. The total strain in material is as follows $[25,26]$ :

$$
\begin{aligned}
\varepsilon= & \left(\frac{1}{E_{2}}+\frac{4 \pi \Omega \Lambda_{d p} h^{2} R(1-\nu)}{G}\right) \sigma \\
& -\left(\frac{E_{3}}{2 E_{2}}+\frac{8 \pi^{2} \Omega \Lambda_{d p} h^{3} R^{2}(1-\nu)^{2}}{G^{2} b}\right) \sigma^{2} \\
& +\frac{64 \pi^{3} \Omega \Lambda_{d p} h^{4} R^{3}(1-v)^{3}}{G^{3} b^{2}} \sigma^{3}+O\left(\sigma^{4}\right) .
\end{aligned}
$$

Assuming specimen under a quasistatic loading, the exciting transducer creates a small oscillatory stress $\Delta \sigma$ of amplitude to the static stress $\sigma$. An additional strain $\Delta \varepsilon$ is created in response to the change in stress $\Delta \sigma$. It can be written as

$$
\begin{aligned}
\Delta \sigma= & \frac{\partial \sigma}{\partial \varepsilon} \Delta \varepsilon+\frac{1}{2} \frac{\partial^{2} \sigma}{\partial \varepsilon^{2}}(\Delta \varepsilon)^{2}+\frac{1}{6} \frac{\partial^{3} \sigma}{\partial \varepsilon^{3}}(\Delta \varepsilon)^{3}+\cdots \\
= & \left(\frac{\partial \varepsilon}{\partial \sigma}\right)^{-1} \Delta \varepsilon+\frac{1}{2} \frac{\partial^{2} \varepsilon}{\partial^{2} \sigma}\left(\frac{\partial \varepsilon}{\partial \sigma}\right)^{-3}(\Delta \varepsilon)^{2} \\
& +\frac{1}{6} \frac{\partial^{3} \varepsilon}{\partial \sigma^{3}}\left(\frac{\partial \varepsilon}{\partial \sigma}\right)^{-4}(\Delta \varepsilon)^{3}+\cdots
\end{aligned}
$$

The third order nonlinear coefficient $\delta_{d p}$ is

$$
\begin{aligned}
\delta_{d p} & =\frac{\partial^{3} \varepsilon}{\partial \sigma^{3}}\left(\frac{\partial \varepsilon}{\partial \sigma}\right)^{-3} \\
& =\frac{384 \pi^{3} \Omega \Lambda_{d p} h^{4} R^{3}(1-\nu)^{3} / G^{3} b^{2}}{\left(1 / E_{2}+4 \pi \Omega \Lambda_{d p} h^{2} R(1-\nu) / G\right)^{3}}+O(\sigma) .
\end{aligned}
$$

In most cases

$$
\frac{1}{E_{2}} \gg \frac{4 \pi \Omega \Lambda_{d p} h^{2} R(1-\nu)}{G} .
$$

Equation (11) can be written as

$$
\delta_{d p}=\frac{384 \pi^{3} \Omega \Lambda_{d p} h^{4} R^{3}(1-v)^{3} E_{2}^{3}}{G^{3} b^{2}} .
$$

\section{Experimental Materials and Methods}

3.1. Materials. The experimental material is GH4169 superalloy. The hardness is $81 \mathrm{HRB}$, and the yield limit is $365 \mathrm{MPa}$. The dimension of specimens is shown in Figure 3 in tensile testing. The dimension of specimens is $200 \mathrm{~mm} \times 50 \mathrm{~mm} \times$ $2 \mathrm{~mm}$ in fatigue bending testing.

3.2. Nonlinear Experimental System. Figure 4 shows the ultrasonic nonlinear testing system. It concludes RITEC advance measurement system, Tektronix DPO 4104B digital phosphor oscilloscope, exciting and received transducers, and computer. The center frequency of exciting transducer is $2.5 \mathrm{MHz}$ and the one of received transducer is $5 \mathrm{MHz}$. 
TABLE 1: Parameters for theoretical models.

\begin{tabular}{lccccccccc}
\hline Number & 1 & 2 & 3 & 4 & 5 & 6 & 7 & 8 & 9 \\
Stress $(\mathrm{MPa})$ & 0 & 50 & 100 & 150 & 200 & 250 & 300 & 350 & 400 \\
$\Lambda_{m p}\left(\times 10^{14} \mathrm{~m}^{-2}\right)$ & 8.64 & 2.14 & 1.87 & 1.56 & 1.28 & 1.01 & 0.72 & 0.44 & 0 \\
$\Lambda_{d p}\left(\times 10^{14} \mathrm{~m}^{-2}\right)$ & 0.45 & 0.45 & 0.52 & 0.83 & 1.11 & 1.39 & 1.68 & 1.96 \\
\hline
\end{tabular}

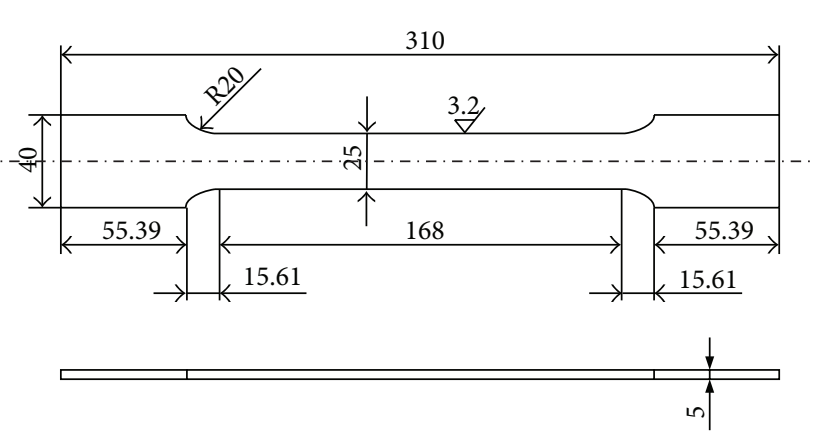

FIGURE 3: Dimensions of specimens of tensile testing.

RITEC advance measurement system generates the exciting wave (shown in Figure 5). It is sinusoidal tone burst of single frequency $2.5 \mathrm{MHz}$. The interactions between the ultrasonic wave and the specimen make wave distort and emerge nonlinearity. The received wave is shown in Figure 6. The odd harmonics show much larger amplitude than the even harmonics in Figure 6(b). Ren et al. also found that the third order acoustic nonlinear parameter has higher sensitivity than the second order one $[27,28]$.

\section{Results and Discussion}

4.1. Effect of Propagation Distance on Relative Nonlinear Coefficient $\beta^{\prime}$. The relative nonlinear coefficients vary with the distance $d$ of transducers. In order to get the optimal distance, measurements are taken at varying propagation distances $d$ (shown in Figure 4) on the intact specimen. Measurements consist of 12 individual measurements at propagation distances that vary from $30 \mathrm{~mm}$ to $140 \mathrm{~mm}$ in increments of $10 \mathrm{~mm}$. In order to make the measurements comparable, the position of the exciting transducer is placed in the same location on the specimens. The experimental curve of relative nonlinear coefficient $\beta^{\prime}$ versus distance is shown in Figure 7. The relative nonlinear coefficient $\beta^{\prime}$ increases monotonously with the propagation distance. When the propagation distance is $90 \mathrm{~mm}$, the relative nonlinear coefficient $\beta^{\prime}$ no longer increases due to attenuation (caused by absorption, diffraction, and scattering).

4.2. Tensile Testing. WDW-E200 universal tensile tester is used to load specimens. Measurements consist of 21 individual measurements at stress levels that vary from $0 \mathrm{MPa}$ to $400 \mathrm{MPa}$ in increments of $20 \mathrm{MPa}$. The curve of loading stress and time is shown in Figure 8.
The experimental curves of relative nonlinear coefficients $\beta^{\prime}$ and $\delta^{\prime}$ versus stress are shown in Figure 9. The error bars are obtained by repeating the measurements three times for each specimen at a certain stress level. The relative nonlinear coefficients $\beta^{\prime}$ and $\delta^{\prime}$ increase with stress when tensile stress is lower than $240 \mathrm{MPa}$. And they both decrease with stress when tensile stress is higher than $240 \mathrm{MPa}$. The length of specimen increases by $0.1 \mathrm{~mm}$ after being loaded to $240 \mathrm{MPa}$. Therefore the plastic deformation begins to exist when tensile stress reaches about $240 \mathrm{MPa}$ which is $65.8 \%$ of the yield strength. For the GH4169 superalloy at room temperature, when the plastic deformation occurs $(\sigma>240 \mathrm{MPa})$, the dislocation pins break and the chord length increases. The dipole dislocation density increases with the stress $[19,26]$. They cause the nonlinear coefficients decrease or changes irregularly with the tensile stress when the tensile stress is higher than $240 \mathrm{MPa}$. So the nonlinear coefficients are closely related to the plastic deformation.

Figure 9(a) shows the relationship of the second order relative nonlinear coefficient $\beta^{\prime}$ versus stress of the experimental data and that predicted by theoretical models. In the models, elastic coefficient $E_{2}=200 \mathrm{MPa}$, shear modulus $G$ $=77.2 \mathrm{MPa}$, Burgers vector $b=25 \mathrm{~nm}$, Poisson ratio $\nu=0.3$, the distance of dislocation $h=20 \mathrm{~nm}$, the dislocation length $L=25 \mathrm{~nm}, a_{1}=0.85, a_{2}=0.15$, and other parameters are shown in Table 1 . The variation trend of experimental data is approximately in agreement with the predicted data by both the monopole model and the comprehensive model. But the experiment data is much closer by the comprehensive model than that by the monopole model.

Figure 9(b) shows the relationship of the third order relative nonlinear coefficient $\delta^{\prime}$ versus stress of the experimental data and that predicted by theoretical model. Because there is no stress value in (13), the theoretical model is not in good agreement with the experimental data.

4.3. Fatigue Bending Testing. The variation trends of ultrasonic nonlinear coefficients and harmonic amplitudes are observed in different fatigue cycles during fatigue bending testing. In order to search the influence of fatigue cycles on nonlinear phenomenon, measurements are taken at varying fatigue cycles. Measurements consist of many individual measurements at fatigue cycles that vary from $0 \mathrm{~N}$ to fracture in increments of $1000 \mathrm{~N}$. The error bars are obtained by repeating the measurements three times for each specimen at certain fatigue cycles. The experimental curves of relative nonlinear coefficient $\beta^{\prime}$ and $\delta^{\prime}$ versus fatigue life are shown in Figure 10. The relative nonlinear coefficients $\beta^{\prime}$ and $\delta^{\prime}$ increase when the fatigue life is lower than $53.3 \%$. The relative 


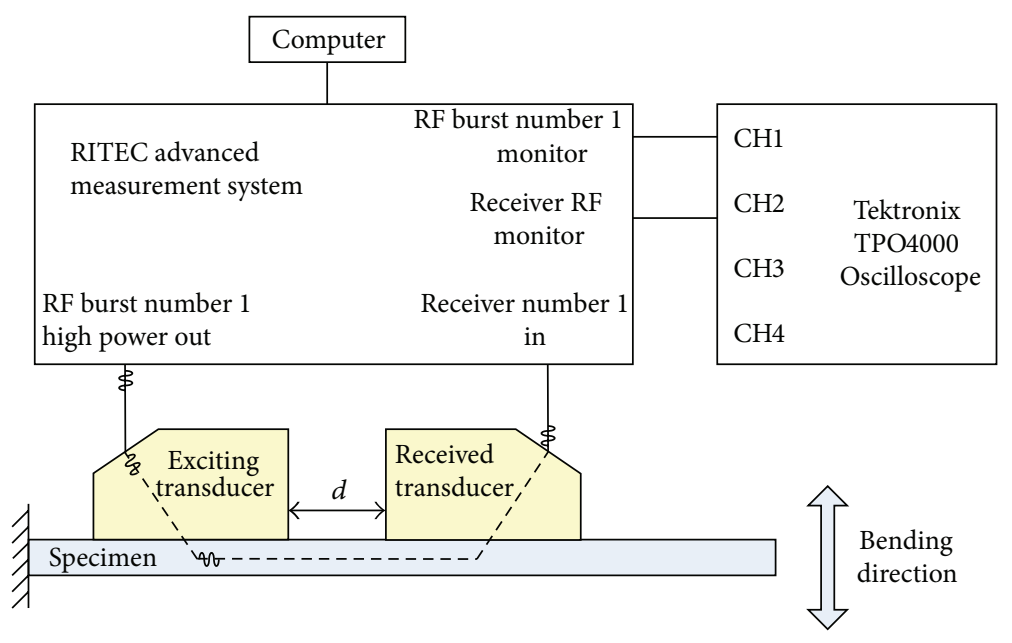

FIGURE 4: Block diagram of ultrasonic nonlinear testing system.

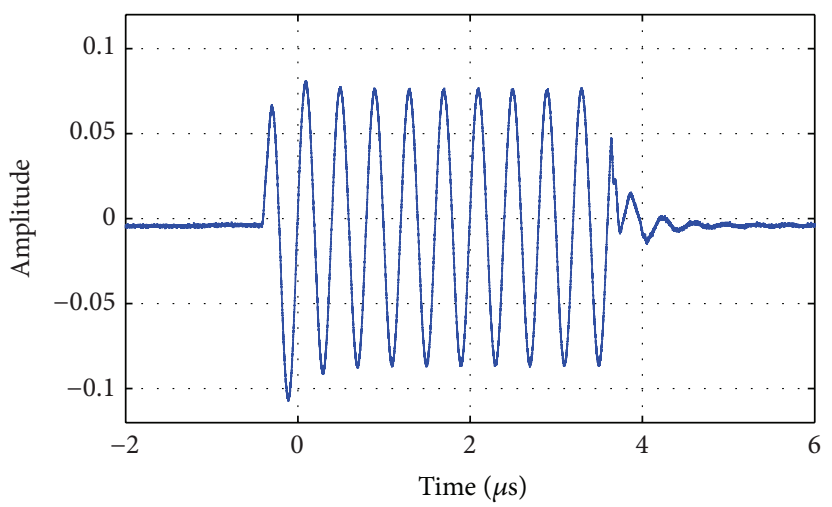

(a) Time domain diagram

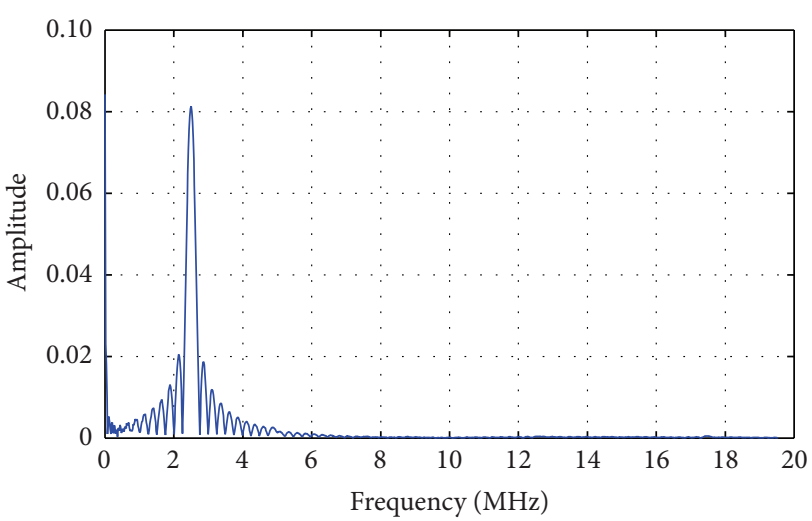

(b) Amplitude frequency diagram

Figure 5: Time domain and amplitude frequency diagrams of exciting signal.

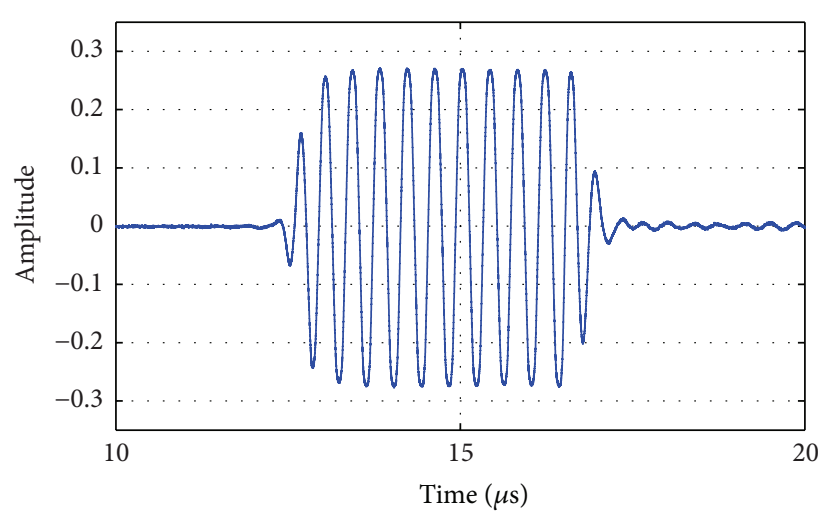

(a) Time domain diagram

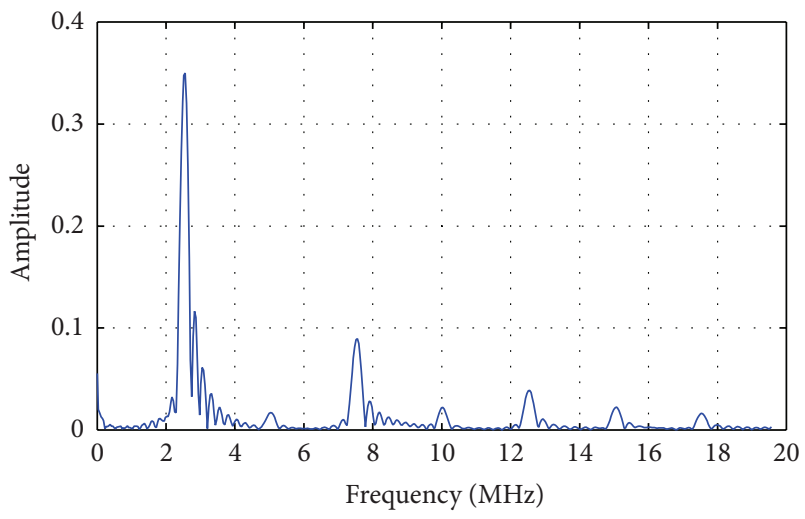

(b) Amplitude frequency diagram

Figure 6: Time domain and amplitude frequency diagrams of received signal. 
TABle 2: Parameters for theoretical models.

\begin{tabular}{lccccccccc}
\hline Number & 1 & 2 & 3 & 4 & 5 & 6 & 7 & 8 & 9 \\
Fatigue life (\%) & 0 & 12 & 24 & 36 & 48 & 60 & 72 & 84 & 100 \\
$\Lambda_{m p}\left(\times 10^{14} \mathrm{~m}^{-2}\right)$ & 8.24 & 2.04 & 1.67 & 1.51 & 1.08 & 0.91 & 0.72 & 0.42 & 0 \\
$\Lambda_{d p}\left(\times 10^{14} \mathrm{~m}^{-2}\right)$ & 0.48 & 0.50 & 0.54 & 0.93 & 1.31 & 1.59 & 1.76 & 2.01 \\
Stress $(\mathrm{MPa})$ & 0 & 16.35 & 28.71 & 35.02 & 42.03 & 44.37 & 46.7 & 49.50 & 51.37 \\
\hline
\end{tabular}

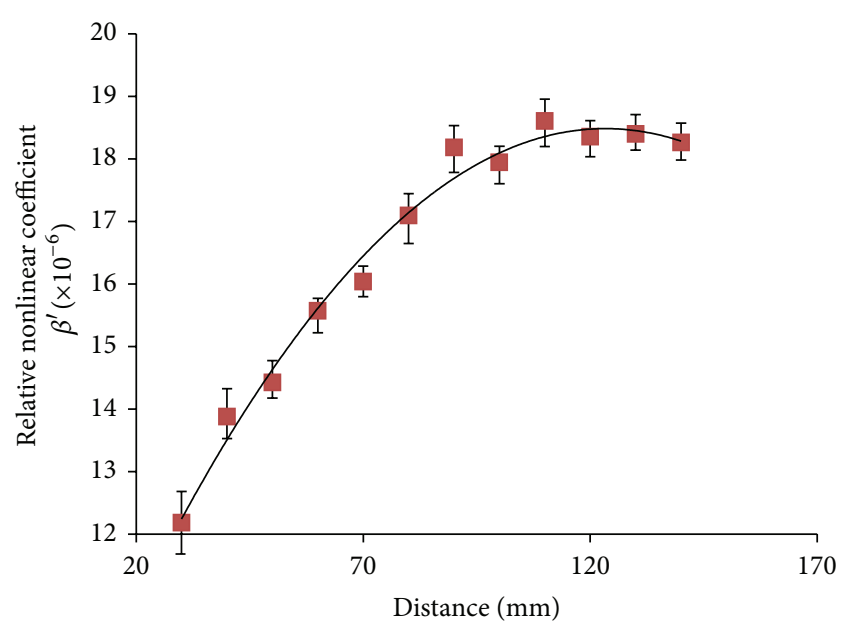

FIGURE 7: Variation in relative nonlinear coefficient $\beta^{\prime}$ as a function of propagation distance.

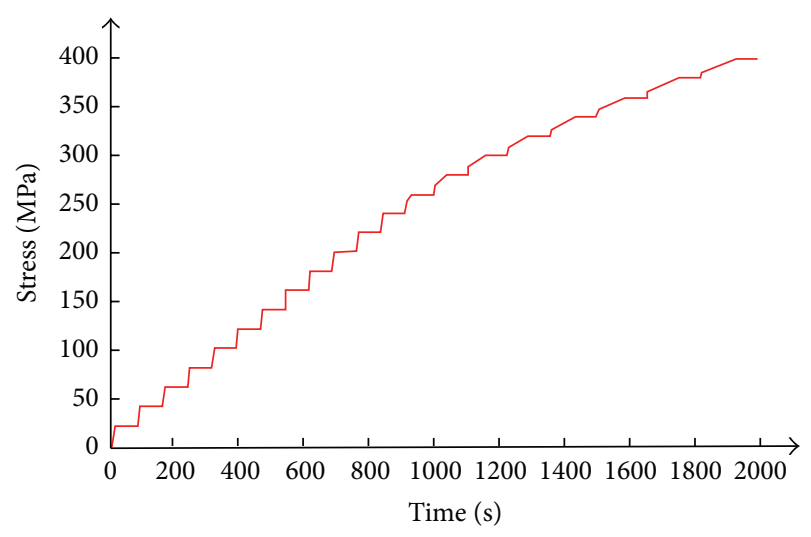

Figure 8: Stress versus time.

nonlinear coefficients $\beta^{\prime}$ and $\delta^{\prime}$ decrease when the fatigue life is higher than $53.3 \%$.

Figure 10(a) shows the relationship of the second order relative nonlinear coefficient $\beta^{\prime}$ versus fatigue life of the experimental data and that predicted by theoretical model. In the models, the parameters are shown in Table 2, and other parameters are the same as the models in Figure 9. The stresses are measured by BIT stress testing. The experimental data is approximately in agreement with the predicted data by the comprehensive model.
Figure 10(b) shows the relationship of the second order relative nonlinear coefficient $\delta^{\prime}$ versus fatigue life of the experimental data and predicted by theoretical model. $\delta^{\prime}$ predicted by the theoretical data increases with the fatigue life, but there is an extreme value in the experimental data. The reason is that only dislocation is considered and no stress value is considered in theoretical model.

In elastic stage, the interior microstructure change with the fatigue cycles, the changes of monopole, and dipole dislocation cause the rising of ultrasonic nonlinearity. When the plastic deformation emerges, the monopole density decreases and veins and cells increase. They induce the decrease of nonlinear coefficient [25]. Therefore the relationship between nonlinear coefficient and fatigue life can be used to predict the fatigue state of metal components if the relationship is known.

\section{Conclusions}

During tensile testing and fatigue bending testing, microstructures change in superalloy. The monopole and dipole densities change with tensile stress or fatigue cycles. They induce the change of the nonlinear coefficients during the ultrasonic propagation.

Measurements are taken at varying stress. The results show that ultrasonic nonlinear coefficients increase with tensile stress in elastic stage, and they decrease in plastic stage. The relationship of the second order nonlinear coefficient as a function of tensile stress can be explained by the comprehensive model.

Measurements are taken at varying fatigue cycles. The results show that ultrasonic nonlinear coefficients increase with the fatigue cycles in elastic stage, because the monopole and dipole densities change with fatigue cycles. The ultrasonic nonlinear coefficients decrease in plastic stage because the monopole density decreases and veins and walls appear. The relationship of the second order nonlinear coefficient as a function of fatigue life can be explained by the comprehensive dislocation model. Therefore, the variation trend of nonlinear coefficient during fatigue testing can be used to predict the fatigue state of materials if the relationship between the nonlinear coefficient and the fatigue cycles is known.

We promote a model of the third order nonlinear coefficient $\delta$. It is used to predict the relationship between the third order nonlinear coefficient $\delta$ and tensile stress or fatigue life. Having no regard for the stress, the theoretical model does not accord with the experimental data. The future work will engage in finding better models to explain the experimental data. 


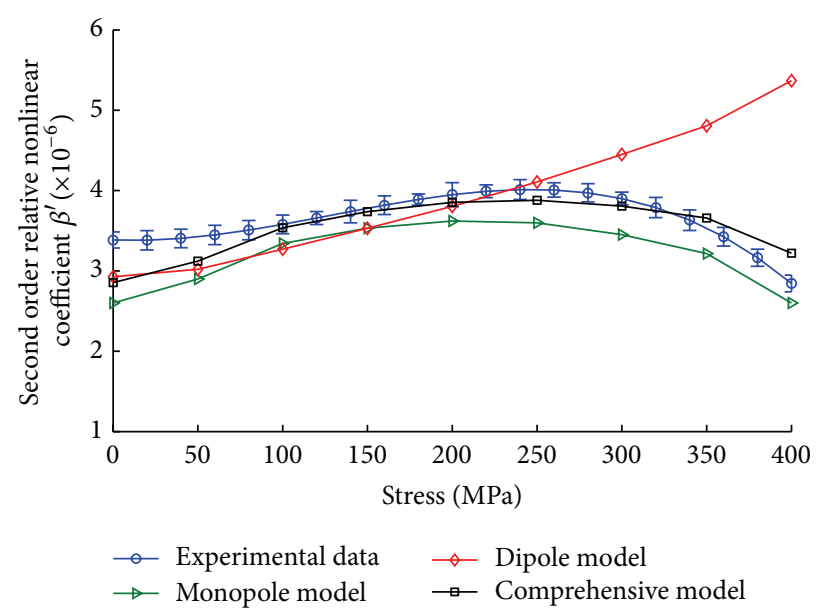

(a) Relative nonlinear coefficient $\beta^{\prime}$

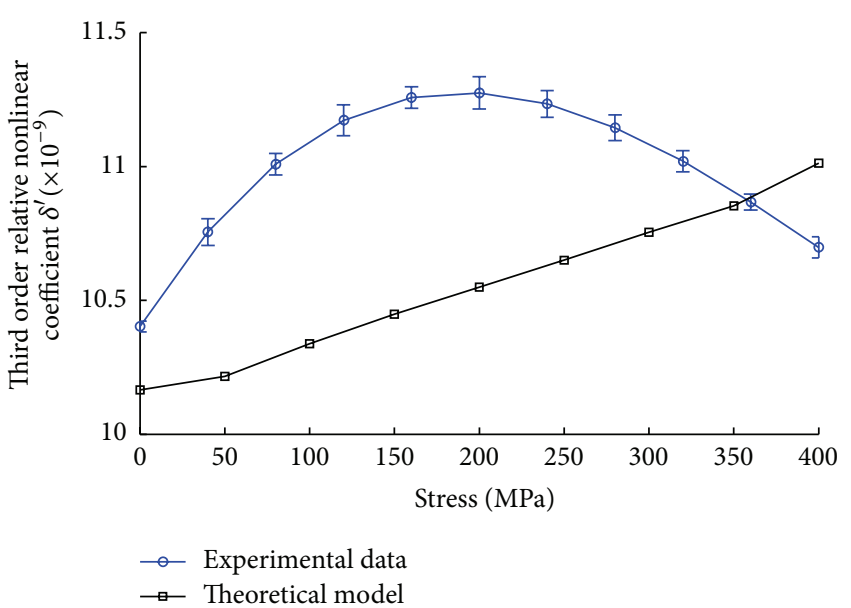

(b) Relative nonlinear coefficient $\delta^{\prime}$

FIGURE 9: Variation in relative nonlinear coefficient $\beta^{\prime}$ and $\delta^{\prime}$ as the function of tensile stress.

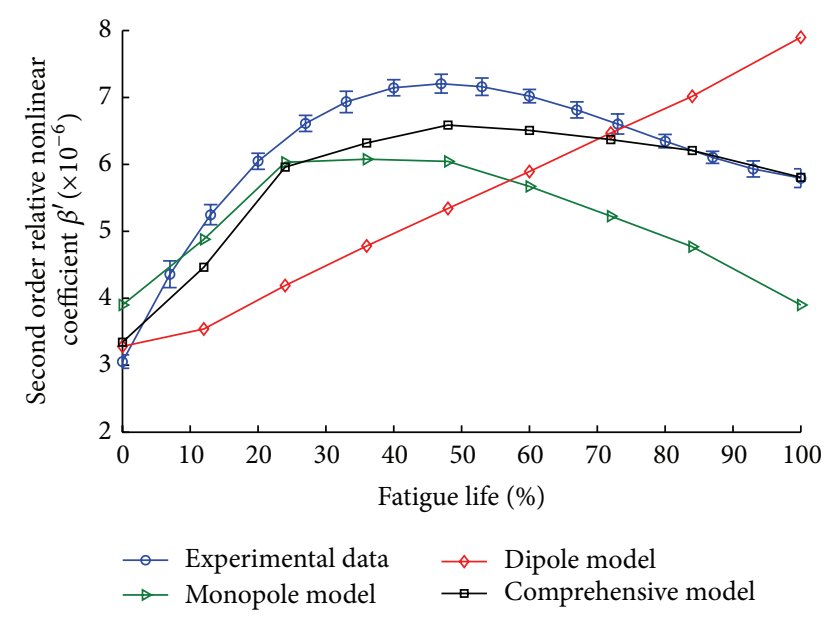

(a) Relative nonlinear coefficient $\beta^{\prime}$

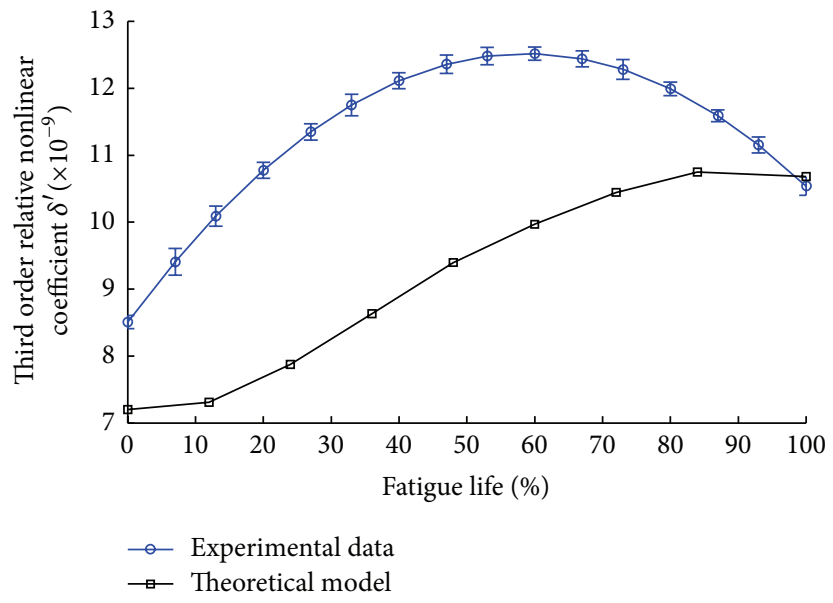

(b) Relative nonlinear coefficient $\delta^{\prime}$

FIGURE 10: Variation in relative nonlinear coefficients $\beta^{\prime}$ and $\delta^{\prime}$ as the function of fatigue life.

\section{Conflict of Interests}

The authors declare that there is no conflict of interests regarding the publication of this paper.

\section{Acknowledgment}

The research is supported by elastic solid residual stress field in situ acoustic energy control mechanism (no. 51275042), which is financed by NSFC of China.

\section{References}

[1] G. S. Shui, Y. S. Wang, and J. Qu, "Advances in nondestructive test and evaluation of material degradation using nonlinear ultrasound," Advances in Mechanics, vol. 35, no. 1, pp. 52-68, 2005.

[2] B. Wu, B.-S. Yan, and C.-F. He, "Nonlinear ultrasonic characterizing online fatigue damage and in situ microscopic observation," Transactions of Nonferrous Metals Society of China (English Edition), vol. 21, no. 12, pp. 2597-2604, 2011.

[3] H. J. Yan, C. G. Xu, Q. Lin, and H. C. Cai, "Metal surface fatigue detection using nonlinear ultrasonic," Applied Mechanics and Materials, vol. 510, pp. 156-162, 2014.

[4] S. V. Walker, J.-Y. Kim, J. Qu, and L. J. Jacobs, "Fatigue damage evaluation in A36 steel using nonlinear Rayleigh surface waves," NDT and E International, vol. 48, pp. 10-15, 2012.

[5] W. Li, Y. Cho, and J. D. Achenbach, "Detection of thermal fatigue in composites by second harmonic Lamb waves," Smart Materials and Structures, vol. 21, no. 8, Article ID 085019, 2012.

[6] A. A. Shah, Y. Ribakov, and C. Zhang, "Efficiency and sensitivity of linear and non-linear ultrasonics to identifying micro and macro-scale defects in concrete," Materials and Design, vol. 50, pp. 905-916, 2013.

[7] T. Kang, H.-H. Kim, S.-J. Song, and H.-J. Kim, “Characterization of fatigue damage of Al6061-T6 with ultrasound, NDT \& E International, vol. 52, pp. 51-56, 2012. 
[8] Z. Su, C. Zhou, M. Hong, L. Cheng, Q. Wang, and X. Qing, "Acousto-ultrasonics-based fatigue damage characterization: linear versus nonlinear signal features," Mechanical Systems and Signal Processing, vol. 45, no. 1, pp. 225-239, 2014.

[9] L. Bjørnø, "Forty years of nonlinear ultrasound," Ultrasonics, vol. 40, no. 1-8, pp. 11-17, 2002.

[10] H. Du, Research on Nonlinear Acoustic in Biomedicine, University of Science and Technology of China, 2007.

[11] K. Y. Jhang, "Nonlinear ultrasonic techniques for nondestructive assessment of micro damage in material: a review," International Journal of Precision Engineering and Manufacturing, vol. 10, no. 1, pp. 123-135, 2009.

[12] A. Hikata, B. B. Chick, and C. Elbaum, "Effect of dislocations on finite amplitude ultrasonic waves in aluminum," Applied Physics Letters, vol. 3, no. 11, pp. 195-197, 1963.

[13] M. A. Breazeale and D. O. Thompson, "Finite-amplitude ultrasonic waves in aluminum," Applied Physics Letters, vol. 3, no. 5, pp. 77-78, 1963.

[14] R. D. Peters and M. A. Breazeale, "Third harmonic of an initially sinusoidal ultrasonic wave in copper," Applied Physics Letters, vol. 12, no. 3, pp. 106-108, 1968.

[15] O. Buck, W. L. Morris, and J. M. Richardson, "Acoustic harmonic generation at unbonded interfaces and fatigue cracks," Applied Physics Letters, vol. 33, no. 5, pp. 371-373, 1978.

[16] P. B. Nagy, "Fatigue damage assessment by nonlinear ultrasonic materials characterization," Ultrasonics, vol. 36, no. 1-5, pp. 375381, 1998.

[17] J.-Y. Kim, L. J. Jacobs, J. Qu, and J. W. Littles, "Experimental characterization of fatigue damage in a nickel-base superalloy using nonlinear ultrasonic waves," Journal of the Acoustical Society of America, vol. 120, no. 3, pp. 1266-1273, 2006.

[18] A. Metya, M. Ghosh, N. Parida, and S. Palit Sagar, "Higher harmonic analysis of ultrasonic signal for ageing behaviour study of C-250 grade maraging steel," NDT and E International, vol. 41, no. 6, pp. 484-489, 2008.

[19] G. Shui, Y.-S. Wang, and F. Gong, "Evaluation of plastic damage for metallic materials under tensile load using nonlinear longitudinal waves," NDT \& E International, vol. 55, pp. 1-8, 2013.

[20] A. Ruiz, N. Ortiz, A. Medina, J.-Y. Kim, and L. J. Jacobs, "Application of ultrasonic methods for early detection of thermal damage in 2205 duplex stainless steel," NDT and E International, vol. 54, pp. 19-26, 2013.

[21] S. Punnose, A. Mukhopadhyay, R. Sarkar, and V. Kumar, "Characterisation of microstructural damage evolution during tensile deformation of a near- $\alpha$ titanium alloy: effects of microtexture," Materials Science and Engineering A, vol. 607, pp. 476-481, 2014.

[22] J. L. Rose, Ultrasonic Waves in Sollid Media, Cambridge University Press, 1999.

[23] D. T. Zeitvogel, K. H. Matlack, J.-Y. Kim, L. J. Jacobs, P. M. Singh, and J. Qu, "Characterization of stress corrosion cracking in carbon steel using nonlinear Rayleigh surface waves," NDT \& E International, vol. 62, pp. 144-152, 2014.

[24] J. H. Cantrell, Fundamentals and Application of Nonlinear Ultrasonic Nondestructive Evaluation, CRC Press, Boca Raton, Fla, USA, 2003.

[25] W. D. Cash and W. Cai, "Contribution of dislocation dipole structures to the acoustic nonlinearity," Journal of Applied Physics, vol. 111, no. 7, Article ID 074906, 2012.

[26] J. H. Cantrell, "Substructural organization, dislocation plasticity and harmonic generation in cyclically stressed wavy slip metals," Proceedings of the Royal Society A: Mathematical, Physical and Engineering Sciences, vol. 460, no. 2043, pp. 757-780, 2004.
[27] M. Amura, M. Meo, and F. Amerini, "Baseline-free estimation of residual fatigue life using a third order acoustic nonlinear parameter," The Journal of the Acoustical Society of America, vol. 130, no. 4, pp. 1829-1837, 2011.

[28] G. Ren, J. Kim, and K.-Y. Jhang, "Relationship between secondand third-order acoustic nonlinear parameters in relative measurement," Ultrasonics, vol. 56, pp. 539-544, 2015. 

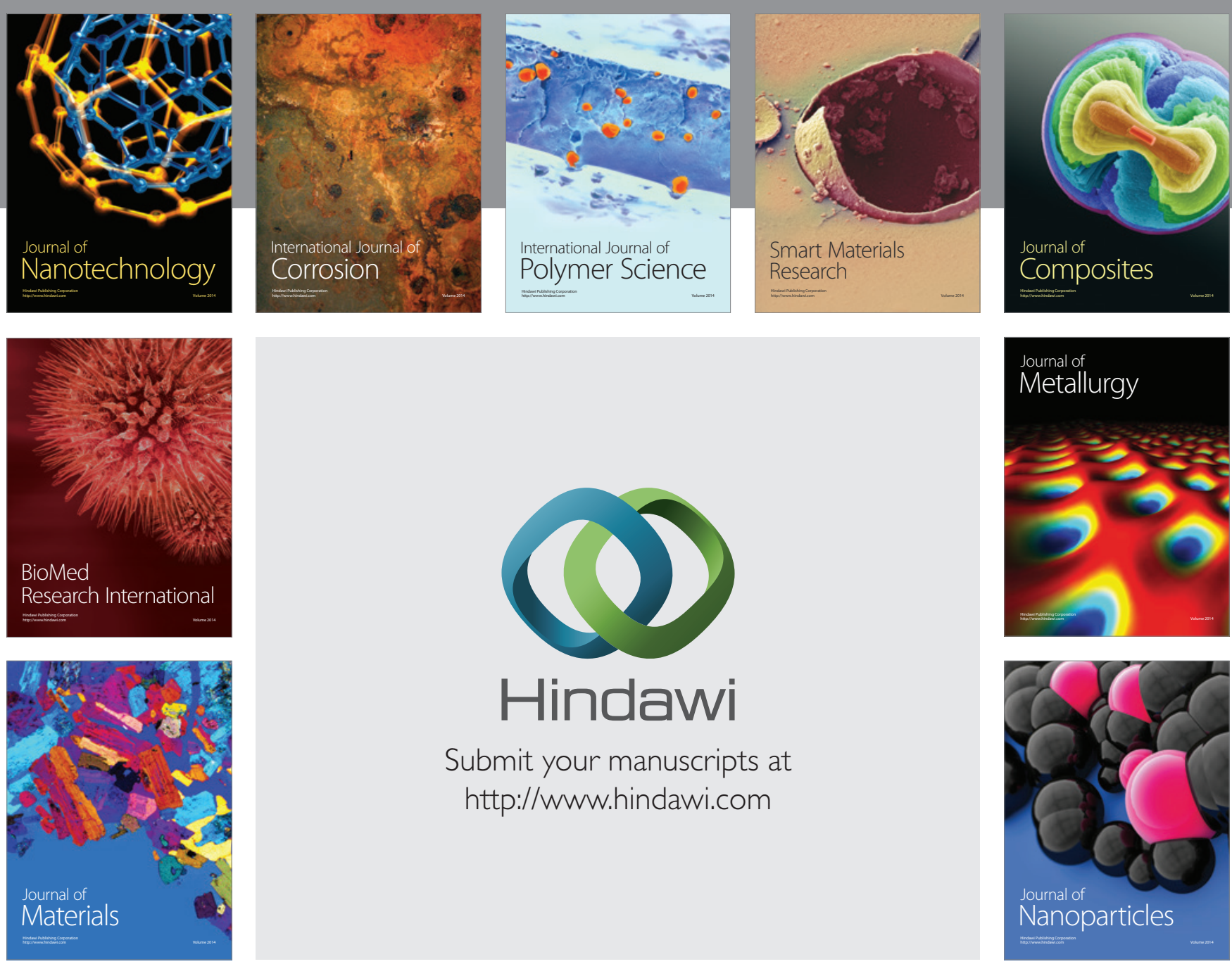

Submit your manuscripts at http://www.hindawi.com
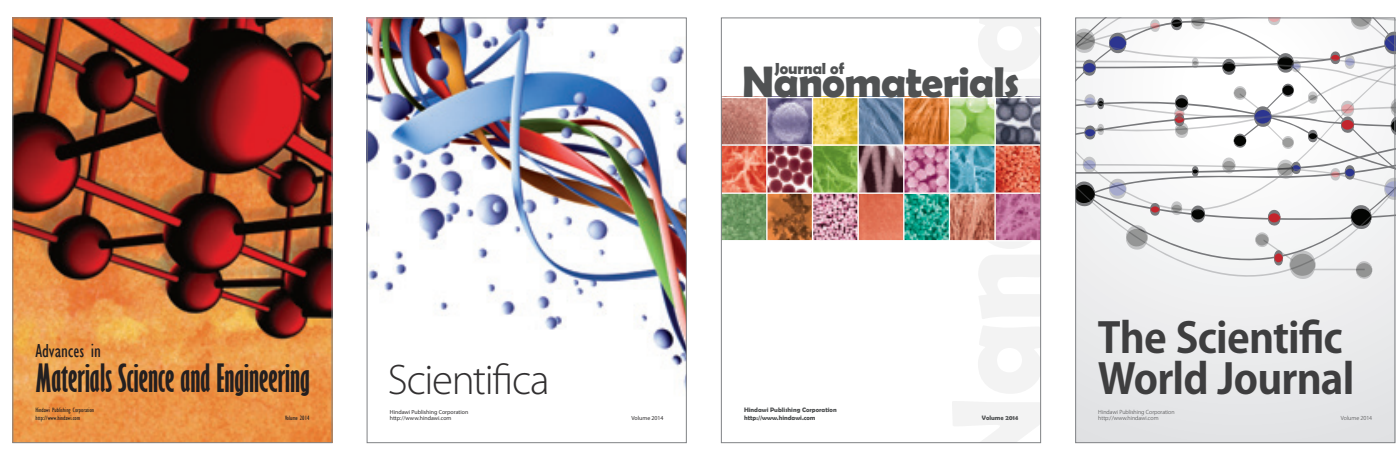

\section{The Scientific World Journal}
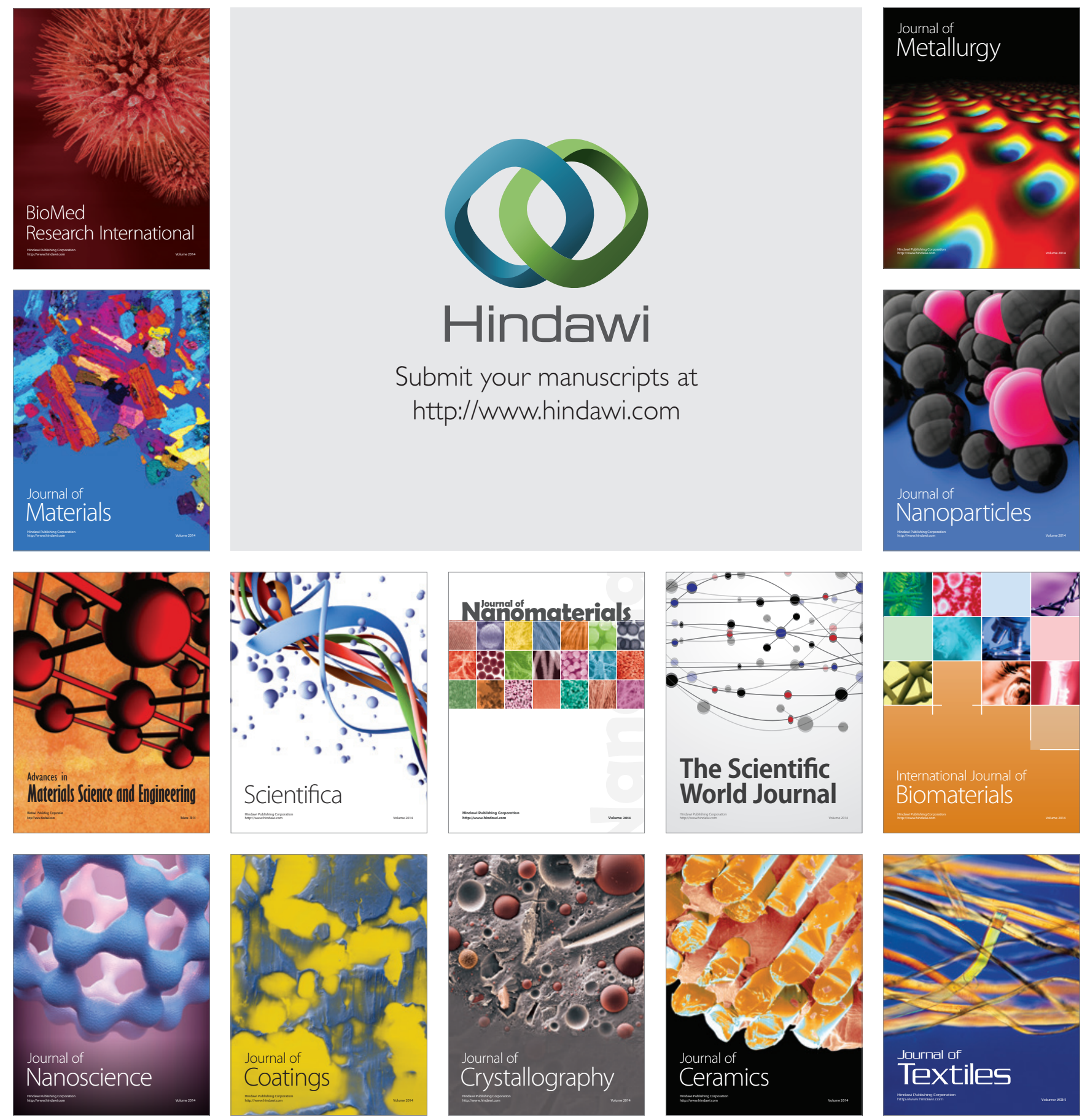